\title{
UNA MIRADA A LOS ESTILOS DE APRENDIZAJE DE LOS ESTUDIANTES DE LA UPTC DESDE LA NOCIÓN DE NATIVO DIGITAL ${ }^{1}$
}

\author{
CLAUDIA ESPERANZA SAAVEDRA BAUTISTA², \\ UNIVERSIDAD PEDAGÓGICA TECNOLÓGICA DE COLOMBIA; \\ JORGE ANDRICK PARRA VALENCIA ${ }^{3}$ UNIVERSIDAD AUTÓNOMA DE BUCARAMANGA
}

Recibido, septiembre 10 de 2014

Concepto evaluación, octubre 07 de 2014

Aceptado, octubre 10 de 2014
Referencia: Saavedra Bautista, C. E., y Parra Valencia, J.A., (2014). "Una mirada a los estilos de aprendizaje de los estudiantes de la UPTC desde la noción de nativo digital". Revista Academia y Virtualidad, 7, (2), 41-52

\section{Resumen}

Este artículo presenta resultados de la investigación "Ambiente virtual de aprendizaje a partir de estilos de aprendizaje en nativos digitales", cuyo objetivo fue identificar los estilos de aprendizaje de mayor predominancia de estudiantes desde el concepto 'nativo digital de la Facultad de Ciencias de la Educación de la Universidad Pedagógica y Tecnológica de Colombia, con el fin de describir sus dimensiones de personalidad dentro de un proceso de aprendizaje $y$, en este sentido, poder ofrecer un ambiente virtual de aprendizaje acorde con sus estilos de aprender. Se trata de una investigación cuasiexperimental con enfoque cuantitativo que se llevó a cabo durante tres semestres consecutivos. El análisis estadístico permite concluir que el estilo de aprendizaje reflexivo presenta una baja predominancia frente a los demás estilos de aprendizaje. Esto sugiere formular prácticas pedagógicas pensadas desde el conocimiento del estudiante, orientadas al fortalecimiento de su capacidad reflexiva.

Palabras clave:aprendizaje, enseñanza, estilo de aprendizaje, dimensión de personalidad, nativo digital.

\section{A LOOK AT THE LEARNING STYLES OF DIGITAL NATIVE UPTC STUDENTS}

\section{Abstract}

This paper presents results of the learning styles of students from higher predominance of the "digital native" concept of the Faculty of Education at the Pedagogical and Technological University of Colombia, in order to describe their

1. Artículo, resultado del proyecto de investigación "Ambiente virtual de aprendizaje a partir de estilos de aprendizaje en nativos digitales" de la UPTC.

2. Especialista Informática para la Docencia; Maestría Tecnología Informática (en curso); Licenciado Informática Educativa, Grupo de Investigación Cetin, Facultad Ciencias de la Educación; Docente Universidad Pedagógica y Tecnológica de Colombia. Correo: claudia. saavedra@uptc.edu.co

3. PhD. Ingeniería Área Sistemas; Magíster Informática; Ingeniero de Sistemas, Grupo de Investigación en Pensamiento Sistémico; Docente Universidad Autónoma de Bucaramanga. Correo: andrick.parra@gmail.com 
personality dimensions within a learning process. It is a quasi - experimental research with quantitative approach was carried out for three consecutive semesters. The statistical analysis indicates that the reflective learning style has a low prevalence compared with other learning styles. This suggests formulate pedagogical practices designed from the student's knowledge, aimed at strengthening their capacity for reflection.

Keywords: learning style, native digital, learning, teaching, personality dimension

\section{UM OLHAR NOS ESTILOS DE APRENDIZADO DOSESTUDANTES DA UPTC DESDE A NOÇÃO DE NATIVO DIGITAL}

\section{Resumo}

Neste artigo se apresentam os resultados da pesquisa "Ambiente virtual de aprendizado a partir de estilos de aprendizado em nativos digitais", cujo objetivo foi a identificação dos estilos de aprendizado mais predominantes nos estudantes desde o conceito de 'nativo digital' da Faculdade de Ciências da Educação da Universidade Pedagógica e Tecnológica de Colômbia, com fim de descrever suas dimensões de personalidade ao interior de um processo de aprendizado e, neste sentido, poder oferecer um ambiente virtual de aprendizado em conformidade com seus estilos de aprendizado. Trata-se de uma pesquisa quase-experimental de enfoque qualitativo desenvolvida durante três semestres consecutivos. A análise estadística permite concluir que o estilo de aprendizado reflexivo apresenta uma baixa predominância frente aos outros estilos de aprendizado. Isto propõe formular práticas pedagógicas pensadas desde o conhecimento do estudante, orientadas ao fortalecimento da sua capacidade reflexiva.

Palavras-chave: aprendizado, ensino, estilo de aprendizado, dimensão de personalidade, nativo digital.

\section{Introducción}

Identificar las diferentes formas de aprender de los estudiantes que están llegando a las aulas universitarias es una labor importante que los docentes encuentran necesario adoptar dentro de sus prácticas pedagógicas para atender las necesidades de aprendizaje de los estudiantes del siglo XXI. Este reconocimiento en cuanto a la forma de aprender amerita proponer estrategias de aprendizaje pensadas desde el interés y el conocimiento del estudiante, con el fin de no convertir el proceso de enseñanza y aprendizaje en una actividad estéril desarrollada en el marco de un currículo descontextualizado. Por esta razón, se ha propuesto como objetivo conocer los estilos de aprendizaje de mayor predominancia de los estudiantes y describir sus dimensiones de personalidad dentro de un proceso de aprendizaje.
La población con la que se llevó a cabo el presente estudio corresponde a estudiantes de la Facultad de Ciencias de la Educación de la Universidad Pedagógica y Tecnológica de Colombia que cursaban la asignatura TIC y Ambientes de Aprendizaje durante el segundo semestre de 2012 y el primero y segundo semestres de 2013. Esta asignatura corresponde al área interdisciplinar del currículo de la Facultad, por lo que los grupos se terminan conformando por estudiantes de diferentes edades, carreras y semestres. La edad de los estudiantes oscila entre los 17 y 30 años.

En síntesis, el trabajo buscó definir los estilos de aprendizaje activo, reflexivo, teórico y pragmático desde la perspectiva del estudiante, del docente, desde la relación entre el estudiante y el profesor, estudiante y estudiante y desde la relación estudiante y las TIC. 
Una mirada a los estilos de aprendizaje de los estudiantes de la UPTC desde la noción de nativo digital

\section{Perspectiva teórica}

Hablar de estilo de aprendizaje se relaciona con el hecho de que cada persona utiliza su propio método o estrategia a la hora de aprender y, aunque las estrategias varían según lo que se quiera aprender, cada persona desarrolla ciertas preferencias o tendencias globales, que definen su estilo de aprendizaje. Gallego y Nevot (2008) afirman que: "Las investigaciones cognitivas han demostrado que las personas piensan de manera distinta, captan la información, la procesan, la almacenan y la recuperan de forma diferente", por lo que resulta oportuno atender a la diversidad cognitiva de los nativos digitales buscando ofrecer ambientes acordes con sus formas de aprender. Según una caracterización de estilos de aprendizaje Keefe (1998), los define como: "rasgos cognitivos, afectivos y fisiológicos, que sirven como indicadores relativamente estables, de cómo los discentes perciben, interaccionan y responden a sus ambientes de aprendizaje"; por otro lado, Dunn y Dunn (1985) definen el concepto estilo de aprendizaje como "la manera en la que [sic] un aprendiz comienza a concentrarse sobre una información nueva y difícil, la trata y la retiene".

Ha sido tanta la importancia que ha tomado el tema de estilos de aprendizaje en contextos formativos que Hoover y Patton (2005) reconocen que "el conocimiento de los estilos cognitivos de aprendizaje ayuda a profesores y estudiantes a comprenderse mejor a sí mismos", de ahí el interés por conocer cómo aprenden los estudiantes que están llegando a las aulas universitarias reconocidos por Prensky (2001) como nativos digitales. Finalmente, Revilla (1999) destaca que los estilos de aprendizaje "son relativamente estables, que aunque pueden cambiar; pueden ser diferentes dependiendo [sic] la situación; son susceptibles de mejorarse; y cuando a los alumnos se les enseña según su propio estilo de aprendizaje, aprenden con más efectividad cognitiva". Para identificar los estilos de aprendizaje se utilizó el test CHAEA $^{2}$ propuesto por García, Gil y Cué (2009).
La metáfora "nativo digital" sin duda ha revolucionado la literatura académica al reconocer en un sujeto capacidades para realizar procesos simultáneos de forma rápida, preferir el lenguaje gráfico al textual y con habilidades para maniobrar de forma ágil un amplio abanico de recursos tecnológicos. Según Prensky (2001), los nativos digitales "nacen en la era digital y son usuarios permanentes de tecnologías con una habilidad consumada, donde su característica principal es sin duda su tecnofilia y su atracción por todo lo relacionado con las nuevas tecnologías"; esta metáfora evidentemente hace alusión a aquellos jóvenes que viven inmersos en un profundo andamiaje tecnológico, donde no navegan sino naufragan en un caos informacional, hecho que desarrolla en ellos otras habilidades como realizar procesos simultáneos e inmediatos donde la velocidad en los procesos resulta un factor importante.

Con el paso del tiempo el autor que propuso esa metáfora ha evolucionado la concepción del "nativo digital", llevándola a una definición que responde a características de la sociedad actual, pensada más en el contexto y la realidad de los sujetos y que denomina "sabiduría digital", donde señala que la capacidad cognitiva de los nativos digitales puede tener un mejoramiento exponencial a través del uso racional e inteligente de las tecnologías de la información y la comunicación. Esta discusión se ha venido posicionando en la literatura educativa como la descripción de un cambio generacional entre docentes y estudiantes, reconocidos como "nativos e inmigrantes digitales".

\section{Método}

La investigación se realizó siguiendo una metodología cuasiexperimental con enfoque cuantitativo, reconociendo esta metodología como aquella que estudia posibles relaciones de causa y efecto, aunque sin seguir condiciones de control riguroso que puedan afectar el experimento.

4. Catalina Alonso y Domingo Gallego. Cuestionario Honey-Alonso de Estilos de Aprendizaje, 1992. [citado el 12-10-13]. Disponible en: http://www. estilosdeaprendizaje.es/chaea/chaea.htm 


\section{Procedimiento}

Teniendo en cuenta que al iniciar el estudio se desconocía el número de estudiantes que llegarían a cursar la asignatura durante el año 2013, se hizo un estimado de la población teniendo en cuenta el número de estudiantes que ingresaron a las TIC y ambientes de aprendizaje durante el segundo semestre de 2012 y revisando los antecedentes del número de inscritos desde la aparición de la asignatura hasta primer semestre del 2012.

Se observó entonces que no se tiene un tamaño de muestra definido para este tipo de estudios; es decir, no hay un diseño muestral explícito en los artículos revisados, aunque a nivel nacional se encontró un artículo en la Universidad Tecnológica de Bolívar titulado "Estilos de aprendizaje y rendimiento académico en estudiantes universitarios", que con una población de 414 estudiantes y luego de realizar un muestreo aleatorio estratificado arrojó una tamaño muestral de 204 estudiantes, pero señalan que por factores externos al estudio se trabajó con un tamaño de muestra de 101 sujetos.

Por lo anterior se decidió realizar una muestra piloto durante el segundo semestre de 2012 utilizando el test CHAEA, con el fin de poder establecer el comportamiento de la variable estilos de aprendizaje en los estudiantes nativos digitales de la facultad de educación en la UPTC; este comportamiento se clasificó en cuanto a la proporción del estilo de aprendizaje predominante. La muestra piloto fue realizada con 50 estudiantes durante la última semana del mes de noviembre del año 2012. La siguiente gráfica ilustra los resultados obtenidos de la muestra piloto.

Según la figura 1, se observa que la categoría con mayor proporción es la de más de un estilo predominante con un $42 \%$ y la de menor proporción es la del estilo de aprendizaje reflexivo con un $4 \%$, por lo que desde que se realizó la muestra piloto la tendencia fue hacia la mayor proporción, la de "más de un estilo predominante", y el estilo "reflexivo", la más baja proporción.

\section{Muestra Piloto}

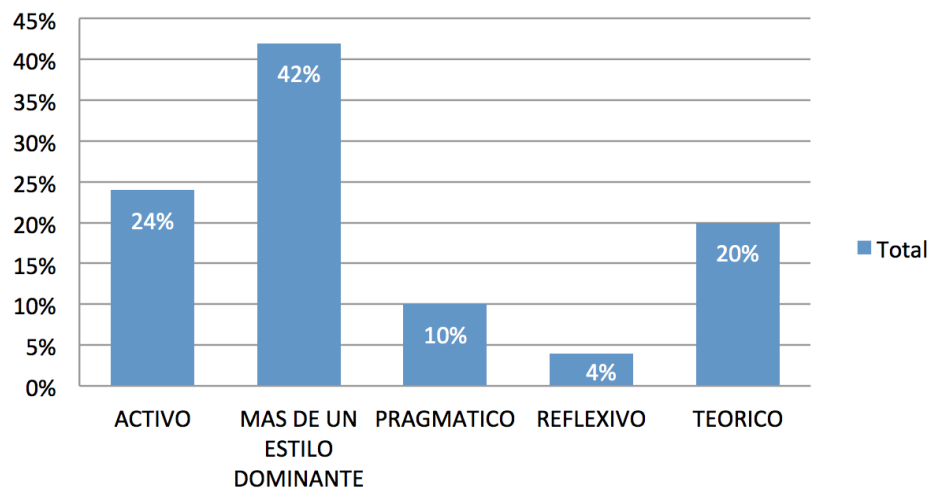

Figura 1. Resultados muestra piloto Fuente: Elaboración propia.

Teniendo en cuenta que el tamaño de una proporción es máxima ${ }^{5}$ cuando $\mathrm{p}=0,5$ y puesto que 0,42 es cercano a este valor, se determinó tomar $\mathrm{p}=0,5$ y como límite para el error de estimación $\mathrm{B}=0,05$, a pesar de que el valor obtenido en la muestra piloto es de $\mathrm{B}=0,1295$ y con un nivel del confianza del 95\%.

\section{Tamaño de muestra}

Una vez realizados los cálculos con la información obtenida de la muestra piloto, el tamaño de muestra sugerido es de alrededor 60 estudiantes; sin embargo, en vista de poder levantar un mayor número de test contestados, se recalculó el tamaño de la muestra ${ }^{6}$ y se logró encuestar a 452 estudiantes.

\section{Instrumentos}

Para identificar los estilos de aprendizaje en los estudiantes se utilizó como instrumento diagnóstico el test CHAEA, el cual se organiza en 80 preguntas y categoriza los estilos de aprendizaje en cuatro variables cognitivas, a saber: activo, reflexivo, teórico y pragmático. Es un test que permite evaluar las dimensiones cognitivas de las personas $\mathrm{y}$ éste ha sido experimentado y contrastado en diversos

5. heaffer and Mendell Hall, p. 100.

6. Para los cálculos se utilizó como probable tamaño de población N=1000 estudiantes puesto que a la fecha no se tenía conocimiento del número de matriculados que ingresarían durante los semestres del desarrollo del estudio. 
ámbitos académicos. Los autores de este test realizan su validación con una muestra de 1.371 estudiantes universitarios de Madrid. Cabe anotar que este test ha tenido una difusión importante y ha sido herramienta de diagnóstico de diferentes investigaciones.

\section{Resultados}

Ahora, los resultados estadísticos de la aplicación del test CHAEA se organizan en cuatro categorías, así: activo, reflexivo, teórico y pragmático. Para los estudiantes que no clasificaron en alguna de estas categorías se propuso la categoría "más de un estilo de aprendizaje", dado que se presentaron estudiantes que desarrollan más de un estilo de aprendizaje, pero se desconoce cómo clasificarlos en estas cuatro dimensiones que propone el test.

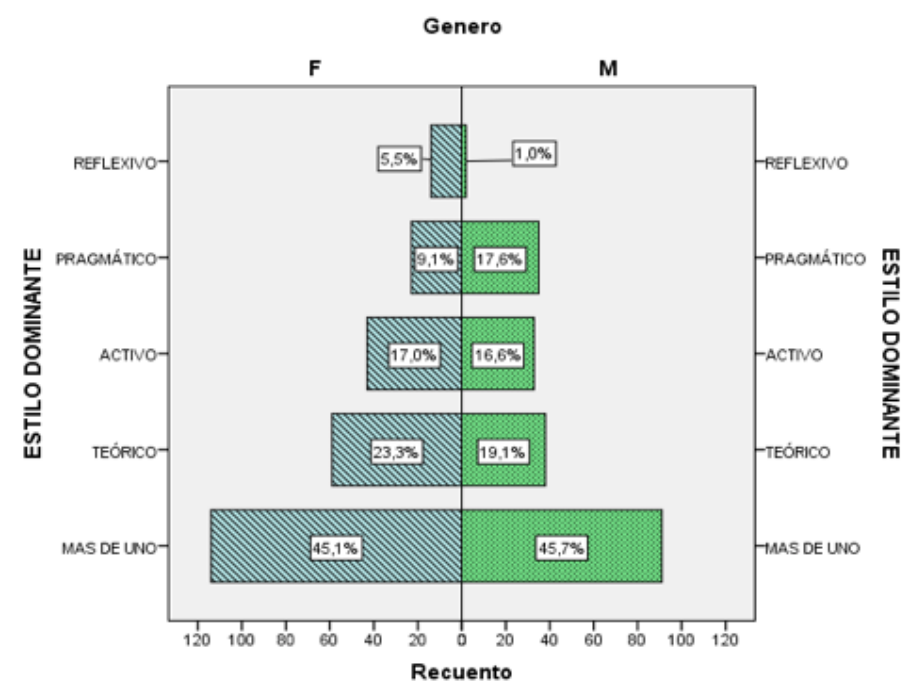

Figura 2. Resultados estilos de aprendizaje versus Género test de CHAEA Fuente: Elaboración propia.

Teniendo en cuenta la figura anterior permite afirmar que de los 452 estudiantes encuestados, únicamente el $5.5 \%$ de las mujeres y el $1 \%$ de los hombres tiene una predominancia al estilo de aprendizaje reflexivo. Lo cual resulta preocupante teniendo en cuenta que aunque los nativos digitales son definidos por la literatura como sujetos capaces de realizar procesos simultáneos de manera rápida, en el afán de los procesos terminan por perder la capacidad reflexiva de los mismos. Del mismo modo con $17,6 \%$, los hombres demuestran tener un estilo de aprendizaje más pragmático en comparación con las mujeres quienes sólo el 9,1\% tienen predominancia por este estilo de aprendizaje.

Para el caso del estilo de aprendizaje activo existe una proporción similar en los resultados de ambos géneros; es decir, en el 17\% de las mujeres hay predominancia por el estilo de aprendizaje activo y en los hombres el 16,6\%. Por otro lado, el test arroja que el 23,3\% de las mujeres es más teórico frente al 19,1\% que se manifiesta en los hombres.

Finalmente, existe una amplia proporción de personas que no se logra categorizar en las dimensiones que propone el test teniendo en cuenta que desarrollan más de un estilo de aprendizaje, pero se desconoce cómo clasificarlas en las cuatro categorías que propone el test.

En la siguiente gráfica se muestra la frecuencia de cada estilo de aprendizaje por semestre, observando que donde mayor se hace el porcentaje de los estilos activo, teórico y la categoría "más de un estilo de aprendizaje" es en los estudiantes de primero y segundo semestres, lo que permite intuir que posiblemente sean los más jóvenes y se ubican en los grupos más numerosos.

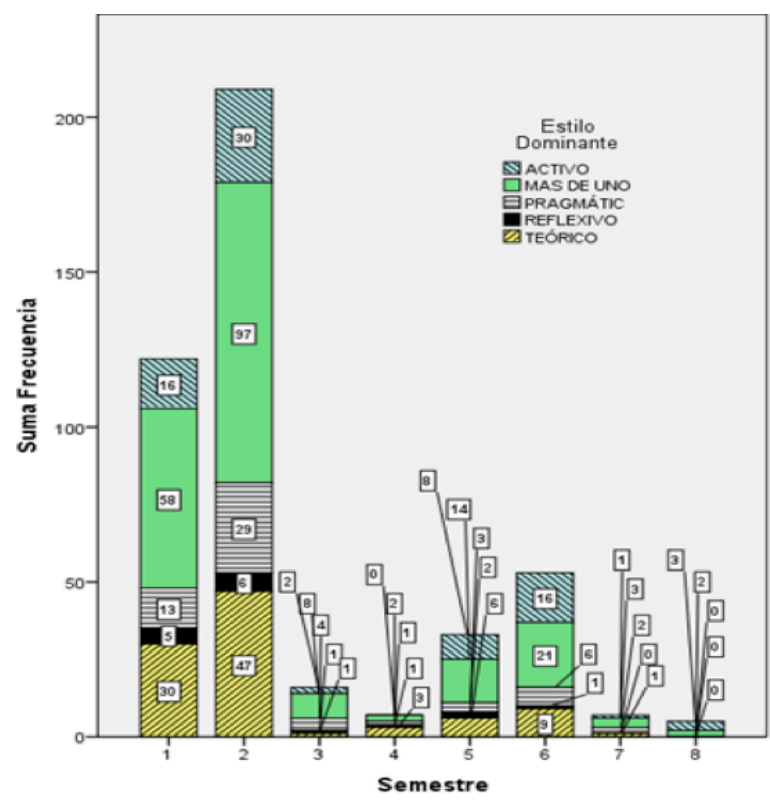

Figura 3. Frecuencia de los estilos de aprendizaje por semestre Fuente: Elaboración propia. 
A continuación se presentan los resultados producto de la relación género, estilo de aprendizaje y diferentes rangos de edad que se encontraron en los datos recogidos.

En la siguiente gráfica se puede observar que los estudiantes en estudio de ambos géneros y con edades entre los 16 y 17 años no presentan predominancia en el estilo de aprendizaje reflexivo. En este rango de edad se puede observar que en el género femenino existe una predominancia en los estilos de aprendizaje activo con un $22 \%$ y teórico con un $26 \%$. Además, el $52 \%$ de este género tiene más de un estilo de aprendizaje. Para el caso del género masculino, el resultado refleja que el $77 \%$ de los hombres entre edades de 16 a 17 años manifiestan tener más de un estilo de aprendizaje.

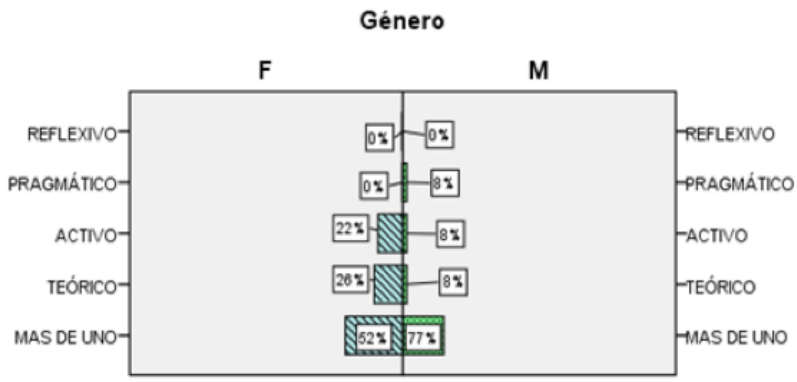

$\vec{\nabla}$

Figura 4. Estilos de aprendizaje de 16 a 17 años Fuente: Elaboración propia.

Para el caso de los estilos de aprendizaje predominantes en los estudiantes cuyas edades oscilan entre los 18 a 20 años, se puede afirmar que los hombres tienden a ser más pragmáticos que reflexivos, mientras que las mujeres en este rango de edad son más reflexivas que pragmáticas. Asimismo, las proporciones que se manejan en cuanto a la predominancia de los estilos de aprendizaje activo y teórico son equivalentes en ambos géneros, la diferencia en porcentaje es mínima, tal como se ilustra en la siguiente figura.

La siguiente gráfica ilustra que entre edades de 21 a 23 años no se reportan hombres con estilo de aprendizaje reflexivo, mientras que en el caso de las mujeres hay un $5 \%$. Además, la figura muestra que los estilos de mayor predominancia en las mujeres son pragmático y teórico, mientras en los hombres es el estilo pragmático.

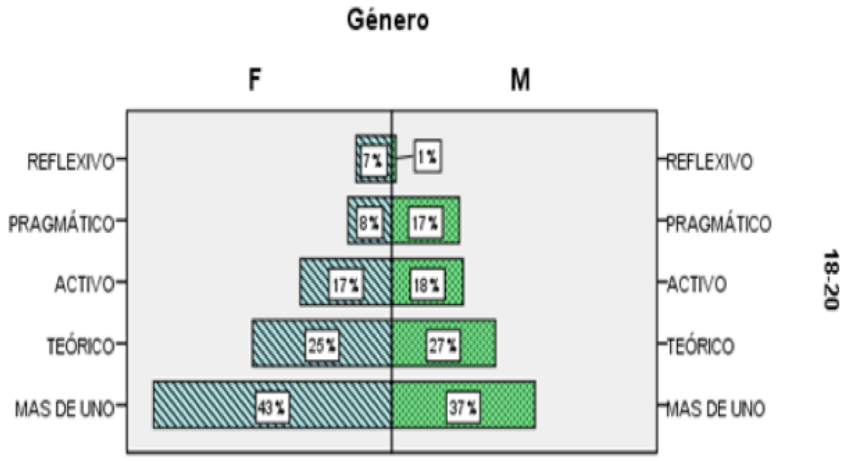

Figura 5. Estilos de aprendizaje de 18 a 20 años Fuente: Elaboración propia.

En hombres y mujeres existe una proporción similar en cuanto al índice de predominancia del estilo activo. Además, más del $50 \%$ de los hombres en este rango de edad clasifican en la categoría "más de un estilo de aprendizaje"; en el caso de las mujeres el $42 \%$.

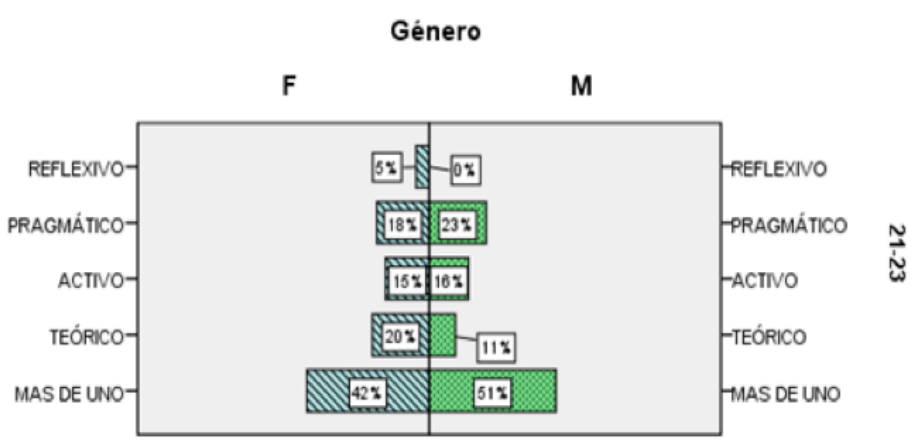

Figura 6. Estilos de aprendizaje de 21 a 23 años Fuente: Elaboración propia.

La siguiente gráfica muestra que a diferencia de las edades previamente valoradas, en el rango de edad entre 24 a 35 años hay una predominancia en mayor porcentaje del estilo de aprendizaje reflexivo en ambos géneros Este dato permite intuir que a mayor edad las personas tienden a ser más reflexivas. Asimismo, los resultados demuestran que más del $50 \%$ de los estudiantes de ambos géneros en este rango de edad presentan más de un estilo de aprendizaje. 
Una mirada a los estilos de aprendizaje de los estudiantes de la UPTC desde la noción de nativo digital

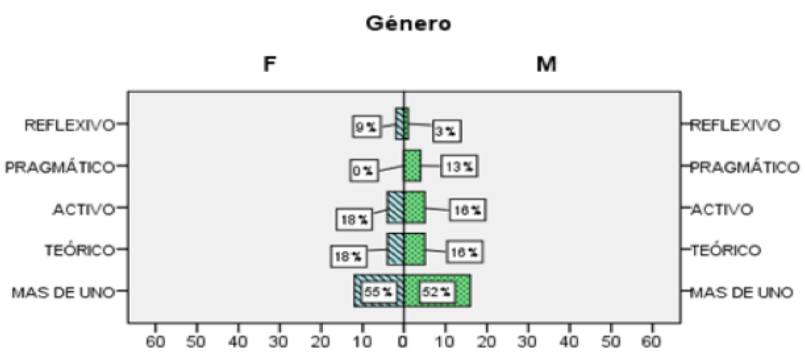

Figura 7. Estilos de aprendizaje de 24 a 35 años Fuente: Elaboración propia.

En la siguiente tabla se resume el porcentaje por semestre de los estilos de aprendizaje predominantes en la población en estudio.

\begin{tabular}{|c|c|c|c|c|c|c|}
\hline \multirow{2}{*}{ Semestre } & \multicolumn{5}{|c|}{ Estilo predominante } & \\
\hline & $\begin{array}{c}\text { MÁS DE } \\
\text { UNO }\end{array}$ & TEÓRICO & Activo & PRAGMÁTICO & REFLEXIVO & \\
\hline 1 & $12,8 \%$ & $6,6 \%$ & $3,5 \%$ & $2,9 \%$ & $1,1 \%$ & $27,0 \%$ \\
\hline 2 & $21,5 \%$ & $10,4 \%$ & $6,6 \%$ & $6,4 \%$ & $1,3 \%$ & $46,2 \%$ \\
\hline 3 & $1,8 \%$ & $0,2 \%$ & $0,4 \%$ & $0,9 \%$ & $0,2 \%$ & $3,5 \%$ \\
\hline 4 & $0,4 \%$ & $0,7 \%$ & $0,0 \%$ & $0,2 \%$ & $0,2 \%$ & $1,5 \%$ \\
\hline 5 & $3,1 \%$ & $1,3 \%$ & $1,8 \%$ & $0,7 \%$ & $0,4 \%$ & $7,3 \%$ \\
\hline 6 & $4,6 \%$ & $2,0 \%$ & $3,5 \%$ & $1,3 \%$ & $0,2 \%$ & $11,7 \%$ \\
\hline 7 & $0,7 \%$ & $0,2 \%$ & $0,2 \%$ & $0,4 \%$ & $0,0 \%$ & $1,5 \%$ \\
\hline 8 & $0,4 \%$ & $0,0 \%$ & $0,7 \%$ & $0,0 \%$ & $0,0 \%$ & $1,1 \%$ \\
\hline & $45,4 \%$ & $21,5 \%$ & $16,8 \%$ & $12,8 \%$ & $3,5 \%$ & \\
\hline
\end{tabular}

Tabla 1. Porcentaje por semestre de estilos de aprendizaje Fuente: Elaboración propia.

Luego de identificar estadísticamente los estilos de aprendizaje de mayor predominancia de la población en estudio y posterior a un proceso de observación en el aula de sus prácticas pedagógicas, en la siguiente tabla se presenta una descripción de los estilos de aprendizaje que propone el test según la experiencia adelantada.

\section{Discusión}

Los resultados obtenidos permiten dar cuenta que un gran porcentaje de los nativos digitales de la facultad de educación de la UPTC tiene predominancia en más de un estilo de aprendizaje, hecho que se asocia a las características que se definen en la literatura por autores como Prensky, Piscitelli y Don Tapscott, quienes afirman que los nativos digitales son sujetos multitareas capaces de desarrollar acciones simultáneas de forma rápida; sin embargo, desde la experiencia adelantada, se puede observar que ante la inmediatez con la que realizan los procesos se termina por descuidar la acción reflexiva de los mismos, afirmación que se soporta con los resultados obtenidos, teniendo en cuenta que la predominancia en el estilo de aprendizaje reflexivo presentó el porcentaje más bajo en los estudiantes encuestados. Un resultado semejante a este estudio arrojó el trabajo realizado en la Universidad Tecnológica de Bolívar por Ruíz y Morales (Estilos de aprendizaje y rendimiento académico en estudiantes universitarios, 2006), el cual explora si existe relación alguna entre los estilos de aprendizaje y el rendimiento académico de los estudiantes universitarios, arrojando como resultado una preferencia hacia el estilo de aprendizaje activo, teórico y pragmático, y un bajo porcentaje en la predominancia del estilo de aprendizaje reflexivo. Asimismo, encontraron que existe una correlación positiva entre el estilo de aprendizaje teórico y el rendimiento académico de los estudiantes, resultado similar al obtenido en este estudio donde se demuestra que en la medida que los estudiantes nativos digitales de la Facultad de Educación de la UPTC sean formados teniendo en cuenta su estilo de aprendizaje, mejorarán su rendimiento académico.

Por otra parte, estudios de similar intención a la investigación adelantada es la experiencia llevada a cabo por Molina (2011), titulada “Análisis de los estilos de aprendizaje de los estudiantes extranjeros y los estilos de enseñanza de los profesores de tres universidades privadas de Puerto Rico", que analiza los estilos de aprendizaje de los estudiantes extranjeros y los estilos de enseñanza de los profesores de tres universidad de Puerto Rico. Esta investigación buscó indagar si existía alguna diferencia por género y nacionalidad en los estilos de aprendizaje de estudiantes y docentes. Dentro de los resultados que arrojó esta investigación se menciona que estadísticamente no existen diferencias significativas entre los estilos de aprendizaje por género, ni por nacionalidad. Asimismo, el estudio comprueba que no hay correspondencia entre el estilo de aprendizaje de los profesores y el estilo de aprendizaje de los estudiantes extranjeros. 


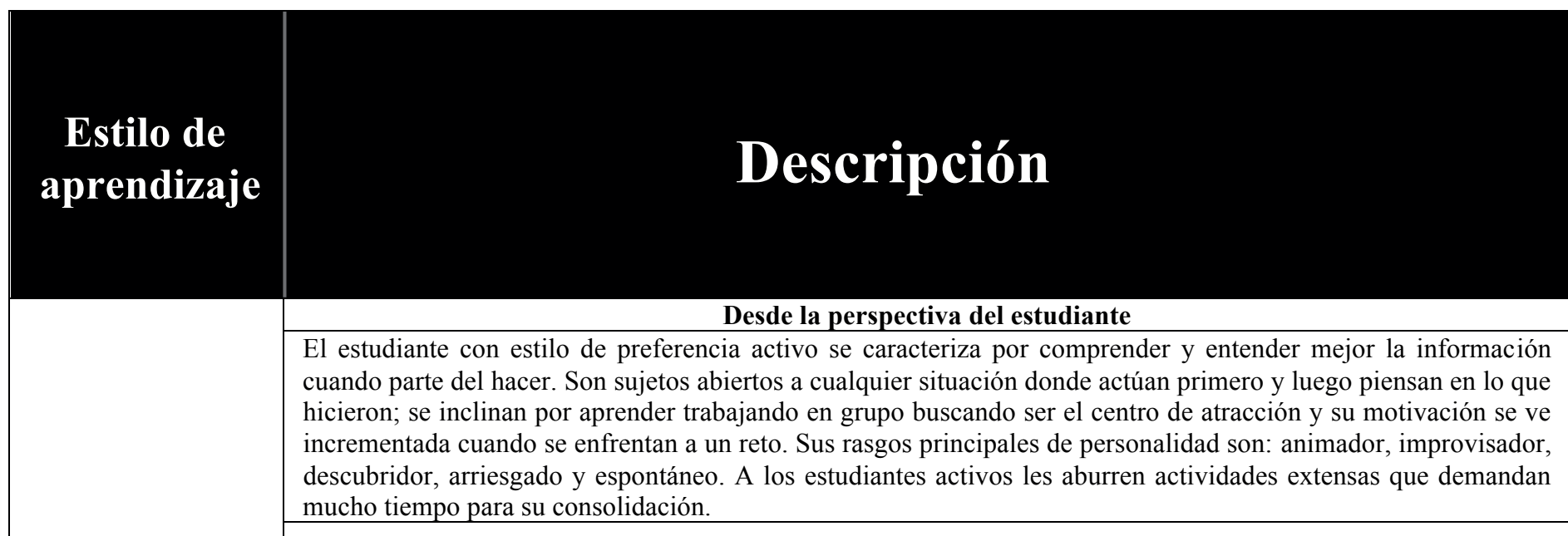

\section{Desde la perspectiva del docente}

El docente percibe al estudiante activo como una persona abierta al conocimiento que disfruta de actividades prácticas, cortas y con resultados inmediatos, donde además los desafíos o retos ante un problema de clase despiertan su motivación e interés.

\section{Relación entre estudiante y docente}

El estudiante activo busca llamar la atención del docente para que promueva prácticas de clase que partan del hacer y del trabajo en grupo, y donde el docente pase de instructor de técnicas a un orientador de los estudiantes.

\section{Relación entre estudiante y estudiante}

La relación entre estudiantes activos está dada en términos de cooperación y trabajo grupal donde prima un ambiente participativo y competitivo que se nutre a partir de la generación de ideas por parte de los integrantes del equipo de trabajo.

\section{Relación entre estudiante y TIC}

El estudiante activo ve en las TIC la posibilidad de ampliar su saber de forma innovadora y espera que el docente promueva estrategias mediadas por TIC donde se aproveche el recurso ofrecido por la institución.

\section{Desde la perspectiva del estudiante}

El estudiante con predominancia en el estilo de aprendizaje reflexivo se caracteriza por comprender mejor la información cuando se toma el tiempo para reflexionar antes que actuar. Estos estudiantes prefieren observar las experiencias y analizarlas desde diferentes perspectivas antes de emitir un juicio; hasta que no están al tanto del panorama completo de la situación prefieren no intervenir. Sus rasgos principales de personalidad son: ponderado, concienzudo, receptivo, analítico, exhaustivo.

\section{Desde la perspectiva del docente}

El profesor percibe al estudiante reflexivo como una persona distante que disfruta de prácticas que tengan que ver con análisis y observación. Se concibe como una persona prudente que tiene en cuenta todas las alternativas posibles antes de realizar cualquier acción. Además se le dificulta convertirse en el centro de atención dentro del grupo. 
Una mirada a los estilos de aprendizaje de los estudiantes de la UPTC desde la noción de nativo digital

\section{Relación entre estudiante y estudiante}

La relación entre estudiantes reflexivos no se pudo definir teniendo en cuenta que en uno de los grupos experimentales según los resultados del test no hay ningún estudiante reflexivo y en el otro grupo experimental solamente hubo un estudiante con predominancia en dicho estilo de aprendizaje, dificultando conocer la relación que puede ocurrir entre estudiantes reflexivos.

\section{Relación entre estudiante y TIC}

El estudiante reflexivo ve en las TIC la posibilidad de conocer diferentes formas de acceder al conocimiento y tratan de utilizarlas de manera lógica y ordenada.

\section{Desde la perspectiva del estudiante}

El estudiante con estilo de preferencia teórica se caracteriza por tener un enfoque lógico en la solución de problemas, siguiendo ordenadamente el desarrollo de las etapas hasta llegar a su solución. En el desarrollo de sus actividades de clase, la teoría es un factor importante, la cual se les facilita ordenar, analizar y sintetizar. En su aprendizaje prima la teoría antes que la acción. Se les dificulta aprender de forma práctica y con actividades donde prime la ambigüedad. Sus dimensiones de personalidad son: metódico, lógico, objetivo, crítico y estructurado.

\section{Desde la perspectiva del docente}

El profesor percibe al estudiante teórico como una persona con fuertes hábitos de lectura que le facilitan el aprendizaje utilizando teorías y modelos. Su aprendizaje es sistémico y promueven la generación de ideas dentro del grupo. Además, se le facilita preguntar e indagar sobre los temas planteados en clase y procura estar documentado.

\section{Relación entre estudiante y docente}

El estudiante teórico tiende a mantener un diálogo constante con el docente que le permita demostrar qué tanto domina el tema de clase. El estudiante teórico ve a su docente como un par con el que puede discutir teorías.

\section{Relación entre estudiante y estudiante}

La relación entre estudiantes teóricos en algunos casos puede presentar conflicto por las diferencias de pensamiento producto de su formación teórica; cuando logran ponerse de acuerdo terminan realizando buenos productos.

\section{Relación entre estudiante y TIC}

El estudiante teórico reconoce en las TIC la posibilidad de llegar a las teorías de grandes intelectuales y la forma como estas tecnologías le permiten esquematizarlas, analizarlas e interpretarlas de forma ágil para ser aplicadas a un contexto real.

\section{Desde la perspectiva del estudiante}

El estudiante con predominancia en el estilo de aprendizaje pragmático prefiere la acción antes que la teoría y disfruta poner en práctica las ideas que se le ocurren. Resuelven problemas de manera rápida sin enfrascarse en una discusión de ideas. Sus dimensiones de personalidad son: experimentador, práctico, directo, eficaz y realista.

\section{Desde la perspectiva del docente}

El profesor percibe al estudiante pragmático como una persona que le gusta poner a prueba sus ideas sin importar los resultados y trabajan de forma eficiente y sincronizada cuando relacionan la teoría con la práctica. Son estudiantes que expresan el deseo de hacer alguna actividad si ven a sus compañeros realizándola.

\section{Relación entre estudiante y docente}

El estudiante pragmático invita al docente a proponer actividades prácticas antes que teóricas. Este estudiante quiere demostrar que es un persona propositiva capaz de llevar a la práctica sus ideas para que luego en compañía del docente se analicen los resultados.

\section{Relación entre estudiante y estudiante}




\section{Relación entre estudiante y estudiante}

La relación entre estudiantes pragmáticos se torna interactiva ya que cada integrante del grupo quiere poner sus ideas en práctica inmediatamente se le ocurren. En el grupo buscan resolver los problemas tomando decisiones de manera rápida y sin importar los resultados.

\section{Relación entre estudiante y TIC}

El estudiante pragmático reconoce en las TIC la posibilidad de comprobar e indagar la pertinencia de sus ideas y opta por utilizar estas tecnologías para adquirir conocimientos que puedan ser aplicados en un contexto real. Estos estudiantes ven en las TIC la oportunidad de fortalecer su capacidad creativa ya que sus acciones van orientadas a la práctica.

Tabla 2. Descripción de los estilos de aprendizaje

Fuente: Elaboración propia.

Asimismo se encontró el estudio adelantado Martínez (2004), "Investigación y análisis de los estilos de aprendizaje del profesorado y de sus alumnos del primer ciclo de educación secundaria obligatoria”, que determina los estilos de aprendizaje de alumnos y profesores del primero ciclo de secundaria en colegios públicos y privados. Entre los resultados resalta que en los estudiantes existe mayor predominancia por el estilo de aprendizaje reflexivo sobre el activo y mayor predominancia en el estilo pragmático sobre el teórico; del mismo modo, afirma que las alumnas participan de un estilo de aprendizaje reflexivo más elevado que los alumnos, por lo que refieren que el género influye en el estilo de aprendizaje.

En esta misma línea se encontró un trabajo desarrollado con estudiantes de posgrado por García, Quintanar, Velázquez y Tapias (2012), “Estilos de aprendizaje y estrategias de aprendizaje: un estudio en discentes de postgrado", donde analizan la relación entre estrategias didácticas y estilos de aprendizaje en estudiantes de posgrados. Los resultados de este estudio demuestran que no hay diferencias significativas en las medias de las puntuaciones de cada estilo de aprendizaje.

También aparece una investigación desarrollada por Alonso (2011), "Estudio comparativo de los estilos de aprendizajes del alumnado que inicia sus estudios universitarios en diversas facultades de Venezuela, México y España", que analizan las preferencias en cuanto a los estilos de aprendizaje de los estudiantes de tres instituciones de Educación Superior de tres países: España, México y Venezuela, obteniendo como resultados que los estudiantes de la Universidad Autónoma Chapingo, México, tienen menores promedios en los estilos de aprendizaje activo y reflexivo. Los alumnos de la Escuela Universitaria de Magisterio de Segovia, Universidad de Valladolid (España) obtienen un alto promedio en el estilo de aprendizaje activo, mientras que los alumnos de la Universidad de Los Andes, Táchira, tienen mayor promedio en el estilo de aprendizaje reflexivo, caso contrario a los resultados obtenidos en la presente investigación.

Finalmente, el trabajo propuesto por Gallego y García (2012), "Los estilos de aprendizaje en la formación inicial de docentes", realiza una exploración de los elementos pedagógicos que se deben tener en cuenta en una sociedad del conocimiento y que integran una nueva cultura del aprendizaje. El estudio gira en torno a la preocupación por mejorar el sistema educativo y contribuir a elevar los informes de desempeño educativo de cada país; además, busca impulsar la calidad de formación inicial del docente y señalan la importancia de considerar que una acción práctica concreta comienza por hacer realidad la implementación de la diversidad de los estilos de aprendizaje.

El estudio concluye que las metodologías líderes del siglo XXI deben desarrollarse desde el conocimiento completo 
del estudiante, de lo contrario se corre el riesgo de caer en una actividad estéril.

El trabajo concluye que el $45,1 \%$ de los estudiantes de la Facultad de Educación de la UPTC se pueden definir como estudiantes multitareas con procesos de aprendizaje aleatorios, tal como los define la literatura $y$, en consecuencia, pueden desarrollar más de un estilo de aprendizaje, permitiendo que un mayor número de actividades propuestas por el docente intervengan en su proceso de aprendizaje. Asimismo, el desarrollo de este proyecto permite reconocer que existe una baja predominancia hacia el estilo de aprendizaje reflexivo y, en la medida en que incrementa el índice de edad en la población estudiada, se eleva la preferencia por este estilo de aprendizaje reflexivo.

En este sentido, los resultados de investigación obtenidos constituyen una alerta para los docentes, puesto que hay que buscar estrategias didácticas que ayuden a fortalecer la capacidad reflexiva de los estudiantes.

\section{Agradecimientos}

Los autores expresan su agradecimiento a la Universidad Pedagógica y Tecnológica de Colombia por el escenario brindado para adelantar este proceso investigativo.

\section{Referencias}

Alonso, C.; Gallego, D. y Honey, P. (1992). “Cuestionario Honey Alonso de estilos de aprendizaje. [citado el 04-07-13]. Disponible en: http://aprenditransfer. com.ar/chaea.shtml

(1999). Los estilos de aprendizaje. $5^{\mathrm{a}}$ ed. Bilbao: Mensajero.

Cué, J.L.G. et ál. (2012). "Estilos de Aprendizaje y Estrategias de Aprendizaje: un estudio en discentes de postgrado". En: Journal of Learning Styles, 5(10).

Curet, N.C. (2011). Análisis de los estilos de aprendizaje de los estudiantes extranjeros y los estilos de enseñanza de los profesores de tres universidades privadas de Puerto Rico [Doctoral dissertation, Universidad del Turabo (Puerto Rico)].

Dunn, R.; Dunn, K. \& Price, G. (1985). "Manual: Learning style inventory". Lawrence, KS: Price Systems, 1 .

Emerson, R. (nd). "Social Exchange theory". In: Annual Review of Sociology, pp. 335-362.

Escalante, C. (sf). "El problema y la hipótesis. Módulo 2" En: Serie Aprender a investigar. ICFES, p. 20.

Gallego, D. (2012). "Los estilos de aprendizaje en la formación inicial del docente. Learning styles-in initial teacher". En: Revista Estilos de Aprendizaje, No. 9, Vol. 9, abril de 2012.

Gallego, D. y Alonso, C. (2008). Estilos de Aprender en el siglo XXI.

García, C.M.A.; Gil, D.J.G. \& Cué, J.L.G. (2009). "Cuestionario Honey-Alonso de Estilos de Aprendizaje CHAEA".

Gil, D.J.G. y Diego, M.C.G. (2012). "Los estilos de aprendizaje en la formación inicial del docente". En: Revista Estilos de aprendizaje, 9(9), 4-20.

Gil,D.J.G.,y Luna,A.N.(2008). “Los estilos deaprendizaje y la enseñanza de las matemáticas”. En: Revista Complutense de Educación, 19(1), 95-112.

Hoover, J.J. y Patton, J.R. (2005). "Diferenciación de currículo e instrucción para los estudiantes del idioma inglés con necesidades especiales". En: Intervención en la escuela y la clínica, 40 (4), 231-235.

Keefe, J. (1988). Aprendiendo Perfiles de Aprendizaje. Asociación Nacional de Escuelas Secundarias.

Martínez, P. (2004). "Investigación y análisis de los estilos de aprendizaje del Profesorado y de sus alumnos del primer ciclo de educación Secundaria obligatoria". En: Actas del I Congreso Internacional de Estilos de Aprendizaje. Madrid: UNED. 
Piscitelli, A. (2006). "Nativos e inmigrantes digitales: ¿Brecha generacional, brecha cognitiva, o las dos juntas y más aún?" En: Revista Mexicana de Investigación Educativa, 11(28), 2006.179-185. [citado el 01-08-13]. Disponible en: http://search.proquest.com/ docview/748436861? accountid $=43790$

(2009). "Nativos digitales: Dieta cognitiva, inteligencia colectiva y arquitecturas de la participación". En: Aula XXI. Santillana, 2009.

Piscitelli, A. et ál. (2009). "Inmigrantes Digitales vs. Nativos digitales".

Prensky, M. (2001). Digital Natives, Digital Immigrants From On the Horizon. MCB University Press. . The Emerging Online Life of The Digital Native: What they do differently because of technology, and how they do it.
. (2009). "H. sapiens digitales: De los inmigrantes digitales y nativos digitales a la sabiduría digital". En: Revista de Educación [en línea], 5 (3), 1-9.

Revilla, D. (1999). "Los estilos de aprendizaje". En: Revista Autoeducación, pp.7-10.

Ruíz B., Trillos J. y Morales J. (2006). "Estilos de aprendizaje y rendimiento académico en estudiantes universitarios, Colombia". Universidad Tecnológica de Bolívar.

Tapias, M.G. et ál. (2011). "Estudio comparativo de los estilos de aprendizajes del alumnado que inicia sus estudios universitarios en diversas facultades de Venezuela, México y España". En: Revista de estilos de aprendizaje, 7(7), 35-62. 